Revista Ambiente: Gestão e Desenvolvimento - Volume 11, n.01, Dezembro/2018

ISSN ONLINE:1981-4127

\title{
QUALIDADE AGROINDUSTRIAL DE FRUTOS DE TAPEREBAZEIROS \\ (Spondias mombin L.) CULTIVADOS EM ÁREAS URBANAS DE BOA VISTA, \\ RORAIMA
}

\section{AGROINDUSTRIAL QUALITY OF TAPEREBAZEIROS (Spondias mombin L.) FRUIT CULTIVATED IN URBAN AREAS OF BOA VISTA, RORAIMA}

\author{
Carolina Soares Marques ${ }^{1}$ \\ Pedro Vitor Pereira Guimarães ${ }^{2}$ \\ Maria Fernanda Berlingieri Durigan ${ }^{3}$ \\ Oscar José Smiderle ${ }^{3}$
}

\begin{abstract}
RESUMO: Considerando a necessidade de estudos que possibilitem melhorar o desenvolvimento de mudas obtidas via sexuada de taperebazeiro (Spondias mombin L.), as caracterizações biométricas dos frutos e físico-químicas das polpas podem ser indicativo importante na seleção para o melhoramento vegetal. No presente estudo objetivou-se avaliar características físicas e físico-químicas de frutos de taperebazeiros cultivados em duas áreas urbanas no município de Boa Vista, Roraima, e classificá-las segundo as normas da legislação brasileira vigente (BRASIL, 2000). Os frutos de taperebá foram colhidos após abscisão, em julho de 2017. As variáveis de qualidade avaliadas foram: comprimento, diâmetro e massa fresca de frutos e endocarpos, número de lóculos, número de sementes, rendimentos de endocarpo e polpa, sólidos solúveis, acidez titulável, $\mathrm{pH}$, relação sólidos solúveis:acidez titulável e coloração. Visando comparação e enquadramento legal das polpas, foram adotados os valores mínimos estabelecidos pelos padrões de identidade e qualidade para polpa de taperebá do Ministério da Agricultura, Pecuária e Abastecimento brasileiro. Houve diferença altamente significativa na maioria das variáveis avaliadas entre as áreas de coleta na qualidade pós-colheita dos frutos de taperebazeiros, indicando grande variabilidade entre as áreas. Há diferença significativa na qualidade física e físico-química de frutos de taperebazeiros. As polpas avaliadas estão em conformidade com os padrões de identidade e qualidade para polpa de taperebá.
\end{abstract}

\footnotetext{
${ }^{1}$ Graduanda em Agronomia da Universidade Federal de Roraima (Bolsista CNPq). carolinasoaresmarques@hotmail.com

${ }^{2}$ Doutorando em Biodiversidade e Biotecnologia da Rede de Biodiversidade e Biotecnologia da Amazônia Legal da Universidade Federal de Roraima. pedrovpg@ hotmail.com

${ }^{3}$ Pesquisadores da Empresa Brasileira de Pesquisa Agropecuária (Embrapa Roraima). maria.durigan@embrapa.br; oscar.smiderle@embrapa.br
} 
Palavras-chave: Taperebá; Cajá; Cajazeira; Qualidade fisiológica; Rendimento; Póscolheita.

\begin{abstract}
Considering the need of studies to improve the development of seedlings obtained by taperebazeiro (Spondias mombin L.), the biometric characterization of fruits and physicochemical characteristics of pulps may be an important indicator in the selection for vegetable breeding. The aim of this study was to evaluate the physical and physicochemical characteristics of taperebazeiro fruits grown in two urban areas in the municipality of Boa Vista, Roraima, and to classify them according to the norms of current Brazilian legislation (BRASIL, 2000). The fruits of taperebazeiro were harvested after abscission in July 2017. Quality variables evaluated were: fruit and endocarp length and diameter, fresh fruit mass and endocarp, number of loci, number of seeds, endocarp yield, pulp yield, soluble solids, titratable acidity, $\mathrm{pH}$, ratio solids solubles:titratable acidity and coloring. Aiming to the comparison and legal framework of pulps, the minimum values established by the identity and quality standards for pulp of cajá were adopted. There was a highly significant difference in the majority of the variables evaluated between the collection areas in the post-harvest quality of the fruits of taperebazeiros, indicating large variability between the areas. There is a significant difference in physical and physicalchemical quality of taperebazeiro fruits grown in the urban area of Boa Vista, Roraima. The pulps evaluated comply with the Brazilian legislation in force.
\end{abstract}

Keywords: Taperebá; Cajá; Cajazeira; Physiological quality; Yield; Post-harvest.

\title{
INTRODUÇÃO
}

A riqueza e a variedade das frutas amazônicas, bem como seu sabor exótico, despertaram o interesse das pessoas. O Brasil é o país com a maior biodiversidade do mundo, o que permite acesso às inúmeras espécies frutíferas. Muitas delas são praticamente desconhecidas e, por tal motivo, são pouco exploradas comercialmente (Penna et al., 2008; Mattietto; Lopes; Menezes, 2010). Dentre as diversas espécies frutíferas, destaca-se o taperebá (Spondias mombin L.), também conhecido como cajá, cajarana, e outros nomes populares. É uma frutífera perene, dispersa nas regiões tropicais da América, África e Ásia (Sacramento; Souza, 2009). No Estado de Roraima, os estudos de Duarte et al. (2010) evidenciam que a espécie está presente em todos os municípios de forma espontânea, principalmente em áreas de mata ciliar do ecossistema de savana, o que a torna ainda mais especial, do ponto de vista agroambiental.

Os frutos são utilizados na fabricação de polpas, sucos, picolés, sorvetes, néctares e geleias de excelente qualidade e consideráveis valores nutritivos (Sacramento; Souza, 2009). No entanto, para se obter bons subprodutos dos frutos de taperebazeiros é importante conhecer a qualidade da matéria-prima (frutos). De acordo com Mattietto; Lopes; Menezes (2010), a caracterização de frutos in natura, sejam estes exóticos ou não, 
vem despertando o interesse da comunidade científica, pelo simples fato de se conhecer toda a potencialidade nutricional que os mesmos podem oferecer. Apresenta, no entanto, alguns problemas fitotécnicos relacionados à produção de mudas da espécie como a germinação irregular e distribuída ao longo do tempo (Azevedo; Mendes; Figueiredo, 2004). Os conhecimentos e tecnologias disponíveis ainda não permitem o cultivo em larga escala, sendo a exploração extrativista, em sua grande maioria (Sacramento; Souza, 2009).

Considerando a necessidade de estudos que possibilitem aperfeiçoar o desenvolvimento e seleção de mudas obtidas via propagação sexuada de taperebazeiro (Spondias mombin L.), as caracterizações biométricas dos frutos e físico-químicas das polpas são importantes para o melhoramento vegetal. No presente estudo objetivou-se avaliar características físicas e físico-químicas de frutos de taperebazeiros cultivados em duas áreas urbanas no município de Boa Vista, Roraima, e classificar as polpas de acordo com a legislação brasileira vigente (BRASIL, 2000).

\section{MATERIAL E MÉTODOS}

Os frutos de taperebá utilizados foram colhidos após abscisão na segunda quinzena de julho de 2017, em duas áreas localizadas no município de Boa Vista, Roraima: Centro de Ciências Agrárias da Universidade Federal de Roraima (CCA), nas coordenadas geográficas 2॰52'07', N, 6042’34' W, altitude de $77 \mathrm{~m}$, e Centro de Pesquisa Agroflorestal de Roraima (CPAF-RR), nas coordenadas geográficas $2^{\circ} 45^{\prime} 22^{\prime} \mathrm{N}$, $60^{\circ} 43^{\prime} 51,34^{\prime} \mathrm{W}$ e altitude de $83 \mathrm{~m}$ ). Os frutos foram colhidos de quatro matrizes em CCA e duas matrizes em CPAF-RR, ambas os plantios para fins ornamentais.

Após a coleta de aproximadamente 200 frutos por área, estes foram selecionados e uniformizados manualmente, sendo descartados os atacados por insetos, doenças e/ou com características de má formação e injúrias mecânicas. Selecionaram-se 50 frutos de cada área para caracterização física e físico-química. As determinações da qualidade dos frutos, endocarpos e polpas de taperebá foram realizadas no Laboratório de Pós-colheita e Agroindustrialização da Embrapa Roraima.

As variáveis de qualidade avaliadas foram: comprimento (CF), diâmetro (DF) e massa fresca (MFF) do fruto, comprimento (CE), diâmetro (DE) e massa fresca (MFE) do endocarpo, número de lóculos (NL), número de sementes (NS), rendimento de endocarpo (RE), rendimento de polpa (RP), sólidos solúveis (SS), acidez titulável (AT), pH, relação sólidos solúveis:acidez titulável e coloração [luminosidade $(\mathrm{L})$, cromaticidade $(\mathrm{C})$ e ângulo hue $\left(^{\circ}\right)$.

$\mathrm{CF}, \mathrm{CE}, \mathrm{DF}$ e DE foram mensurados utilizando-se paquímetro digital $(0,01 \mathrm{~mm})$, sendo os valores expressos em milímetros ( $\mathrm{mm})$. MFF e MFE foram mensurados utilizando-se balança analítica $(0,001 \mathrm{~g})$, com valores expressos em gramas $(\mathrm{g})$. NL e NS foram contabilizados a partir de cortes transversais feitos nos endocarpos. RE e RP foram calculados a partir da separação destas partes dos frutos, determinados através das 
respectivas massas, com auxílio de balança analítica, sendo os valores expressos em porcentagem $(\%)$.

As determinações de $\mathrm{AT}$, $\mathrm{SS}$ e $\mathrm{pH}$, foram realizadas de acordo com os métodos indicados pelo IAL (2008), em sala climatizada $\left(25 \pm 1^{\circ} \mathrm{C}\right)$, e os dados, quando necessário, corrigidos a $25^{\circ} \mathrm{C}$. Os terores de SS foram avaliados refratometricamente, utilizando-se refratômetro digital portátil, calibrado com água destilada $\left(0,0{ }^{\circ} \mathrm{Brix}\right)$, com resultados expressos em ${ }^{\circ}$ Brix. A AT foi determinada por titulação volumétrica. Utilizou-se $15 \mathrm{~g}$ de polpa homogeneizada em $300 \mathrm{~mL}$ de água destilada, com auxílio de aparelho tipo mixer. Tomaram-se três réplicas de $100 \mathrm{~mL}$ em erlemayers, as quais foram adicionadas 3 gotas de solução do indicador fenolftaleína. As amostras foram tituladas com solução de hidróxido de sódio $(\mathrm{NaOH})$ a $0,1 \mathrm{~N}$, com os resultados expressos em $\mathrm{g}$ de ácido cítrico/100 g de polpa. O índice de maturação (SS:AT) foi calculado pela relação entre os teores de SS e AT.

Para a determinação de $\mathrm{pH}$ utilizou-se potenciômetro digital, previamente calibrado com soluções tampão em $\mathrm{pH} 4,0$ e 7,0. As leituras foram feitas em extrato obtido misturando-se $30 \mathrm{~g}$ de polpa e $300 \mathrm{ml}$ de água destilada, homogeneizados com auxílio de mixer. O eletrodo do equipamento era submerso no extrato e mantido até estabilização dos valores. A coloração da polpa dos frutos, mensurada em índices de luminosidade ( $\mathrm{L}^{*}$ ), cromaticidade $\left(\mathrm{C}^{*}\right)$ e ângulo hue ou de cor $\left(h^{\circ}\right)$, foi determinada com uma leitura feita diretamente na casca de cada um dos frutos, utilizando-se colorímetro digital Minolta ${ }^{\circledR}$ calibrado em placa padrão ( $\mathrm{Y}=87,2 ; \mathrm{x}=0,3167 ; \mathrm{y}=0,3237)$, e com os resultados expressos segundo o sistema CIE L* a* b*.

Visando comparação e enquadramento legal das polpas, foram adotados os valores mínimos estabelecidos pelos Padrões de Identidade e Qualidade para polpa de cajá (Brasil, 2000). Utilizou-se delineamento experimental inteiramente casualizado, considerando-se as duas áreas (CCA e CPAF) como tratamentos. Para caracterização biométrica adotou-se cinquenta repetições (50 frutos) e, para qualidade agroindustrial das polpas, utilizou-se cinco repetições, contendo polpa de 10 frutos cada. Os dados foram submetidos ao teste de normalidade de Shapiro-Wilk, e quando não apresentaram distribuição normal, foram transformados utilizando a operação matemática $(\sqrt{x}+1)$ para aproximação da curva normal. Posteriormente, todos os dados foram validados estatisticamente por meio de teste $\mathrm{t}$ de Student a 5\% de probabilidade, utilizando o ambiente R, versão 3.4.3 (R CORE TEAM, 2018).

\section{RESULTADOS E DISCUSSÃO}

Os dados obtidos nas avaliações não apresentaram distribuição normal para as variáveis DE, MFE, NL e NS, sendo então necessária a transformação dos dados $(\sqrt{x}+1)$, visando atender os pressupostos do teste estatístico. De acordo com Campos (2000) quando algum dos requisitos para o emprego da estatística paramétrica (normalidade da distribuição) não é preenchido pelos dados da amostra experimental, a transformação dos 
dados é um recurso complementar, destinado a atender os pressupostos dos testes estatísticos.

Constatou-se nos resultados obtidos diferença altamente significativa $(\mathrm{p}<0,001)$ para a maioria das variáveis avaliadas entre as áreas de coleta na qualidade pós-colheita dos frutos de taperebazeiros, indicando grande variabilidade entre as áreas estudadas. Os coeficientes de variação entre 1,42 (L*) e 38,31 (RE) indicam dispersões de dados variando de baixa a alta (Pimentel-Gomes, 2009). Apesar do alto coeficiente de variação (38,31\%), a variável RE apresentou distribuição normal. Não foram observadas diferenças significativas ( $\mathrm{p}>0,05$ ) no $\mathrm{CE}$ e na $\mathrm{L}^{*}$ do pericarpo (Tabelas 1 e 2). Os resultados obtidos na caracterização física dos frutos e endocarpos de taperebazeiros das diferentes áreas estão apresentados na Tabela 1.

Tabela 1. Valores médios de biometria e rendimento de frutos de taperebazeiros cultivados em duas áreas urbanas de Boa Vista, Roraima

\begin{tabular}{cccccc}
\hline \multirow{2}{*}{ Variáveis } & \multicolumn{2}{c}{ Áreas } & & & \\
\cline { 2 - 4 } & CCA & CPAF-RR & Valor de $p$ & Média geral & CV (\%) \\
\cline { 2 - 4 } Comprimento do fruto (mm) & $38,23 \pm 1,84$ & $36,18 \pm 1,65$ & $6,075 \mathrm{e}^{-08 *}$ & 37,21 & 5,43 \\
Diâmetro do fruto (mm) & $22,14 \pm 1,65$ & $24,68 \pm 1,39$ & $5,715 \mathrm{e}^{-13} *$ & 23,41 & 8,49 \\
Massa fresca do fruto (g) & $10,14 \pm 1,59$ & $11,06 \pm 1,22$ & $0,002 *$ & 10,60 & 14,02 \\
Comprimento do endocarpo (mm) & $30,52 \pm 4,23$ & $30,24 \pm 1,47$ & $0,663 \mathrm{~ns}$ & 30,38 & 10,38 \\
Diâmetro do endocarpo (mm) & $15,45 \pm 0,89$ & $18,11 \pm 1,01$ & $0,001 *$ & 16,78 & 2,65 \\
Massa fresca do endocarpo (g) & $2,36 \pm 0,65$ & $4,91 \pm 0,56$ & $0,001 *$ & 3,64 & 7,07 \\
Número de lóculos (un) & $4,8 \pm 0,79$ & $4,4 \pm 0,48$ & $0,001 *$ & 4,57 & 5,87 \\
Número de sementes (un) & $1,6 \pm 0,90$ & $2,2 \pm 0,95$ & $0,001 *$ & 1,92 & 16,44 \\
Rendimento de endocarpo (\%) & $22,49 \pm 5,89$ & $44,90 \pm 6,77$ & $<2,2 \mathrm{e}^{-16 *}$ & 33,69 & 38,31 \\
Rendimento de polpa (\%) & $77,51 \pm 5,89$ & $55,10 \pm 6,77$ & $<2,2 \mathrm{e}^{-16 *}$ & 66,31 & 14,47 \\
\hline
\end{tabular}

$\mathrm{N}=50 ; \mathrm{CCA}=$ Centro de Ciências Agrárias da Universidade Federal de Roraima; CPAF-RR = Centro de Pesquisa Agroflorestal de Roraima; $\mathrm{CV}=$ coeficiente de variação; $*=$ significativo ao nível de $5 \%$ de probabilidade; ns = não significativo ao nível de 5\% de probabilidade.

Os frutos colhidos em CCA são mais alongados do que os colhidos em CPAF-RR, apresentando maior comprimento, com média de 38,23 $\pm 1,84$, enquanto, os frutos de CPAFRR tinham em média $36,18 \pm 1,65 \mathrm{~mm}$ de comprimento. Os frutos de taperebazeiros colhidos em Boa Vista, Roraima, têm comprimento médio semelhante ao de genótipos de cajazeira avaliados por Soares et al. (2006) em Teresina, Piauí, com comprimentos de 
frutos variando de 29,5 a $39,8 \mathrm{~mm}$. Moura et al. (2003) também registraram valores aproximados de comprimento de fruto, com média de $34,5 \mathrm{~mm}$, em experimento de avaliação da evolução do crescimento e da maturação de frutos de cajazeira em Bananeiras, Paraíba. Os frutos de Belém, Pará, avaliados por Mattietto; Lopes; Menezes (2010) para fins de processamento agroindustrial apresentaram variação no comprimento de 15,00 a 48,50 mm, com média geral de 29,30 $\mathrm{mm}$.

Para a variável DF, as amostras oriundas de CPAF-RR apresentaram os maiores valores médios, com 24,68 $\pm 1,39 \mathrm{~mm}$, enquanto as oriundas de CCA tinham em média $22,14 \pm 1,65 \mathrm{~mm}$, indicando que os frutos de taperebazeiros de CPAF-RR são mais largos do que os de CCA. Os valores médios de DF aqui registrados são aproximados aos encontrados na literatura, variando de 18,30 a 26,80 mm (Moura et al., 2003; Azevedo; Mendes; Figueiredo, 2004; Soares et al., 2006; Mattietto; Lopes; Menezes, 2010; Quadros et al., 2015).

A diferença entre as áreas de coleta ficou bem evidenciada na massa individual dos frutos, onde as amostras oriundas de CPAF-RR apresentaram médias de $11,06 \pm 1,22 \mathrm{~g}$, e as de CCA tinham em média 10,14 $\pm 1,59 \mathrm{~g}$, o que representa um acréscimo de $9,1 \%$ de massa fresca. Relaciona-se principalmente a variabilidade geral de plantas de polinização aberta e mudas oriundas de sementes. As massas frescas dos frutos avaliados são semelhantes aos frutos avaliados por Cunha et al. (2011), oriundo de taperebazeiro de Macapá, Amapá, com valores de MFF de 6,6 a 22,7 g, com média de 12,7 g. As MFF aqui avaliadas apresentam menor amplitude do que os frutos avaliados por Mattietto; Lopes; Menezes (2010), com valores variando de $1,35 \mathrm{~g}$ até $16,47 \mathrm{~g}$, com média de $7,19 \mathrm{~g}$.

A variável $\mathrm{CE}$ não apresentou diferença significativa ao nível de $5 \%$ de probabilidade $(\mathrm{p}=0,663)$ entre as áreas estudadas, sendo a média geral de $30,38 \mathrm{~mm}$, valor aproximado aos encontrados na literatura, com valores de CE que variaram de 24,7 mm até 43,2 mm (Azevedo; Mendes; Figueiredo, 2004; Quadros et al., 2015). Já quanto ao DE, a diferença entre as áreas foi significativa novamente. Os frutos oriundos de CPAF-RR apresentaram maiores valores de DE $(18,11 \pm 1,01 \mathrm{~mm})$, do que os frutos de CCA, com média de $15,45 \pm 0,89 \mathrm{~mm}$. Os valores de DE registrados nas duas áreas estudadas são semelhantes aos encontrados na literatura, onde os valores variam de $19,9 \mathrm{~mm}$ até $26,6 \mathrm{~mm}$ (Quadros et al., 2015; Azevedo; Mendes; Figueiredo, 2004).

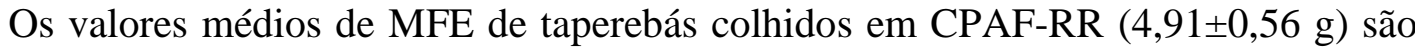
superiores aos encontrados em frutos colhidos em CCA, com média de 2,36 $\pm 0,65 \mathrm{~g}$, correspondendo a um acréscimo de 108,1\%. Os valores médios de MFE de taperebás das duas áreas avaliadas são semelhantes aos encontrados na literatura, com valores variando de 0,51 até 2,42 g (Azevedo; Mendes; Figueiredo, 2004; Quadros et al., 2015). Geralmente, a alta irregularidade de características morfológicas de frutos e endocarpos pode estar associada a diversos fatores. Para Mattietto; Lopes; Menezes (2010) essa irregularidade nas dimensões dos frutos é bastante comum, tendo em vista a região produtora, a forma de cultivo, o clima da região, entre outros fatores que podem afetar as características de desenvolvimento dos frutos. 
Os endocarpos oriundos da área CCA quando comparados com a área CPAF-RR, apresentaram as maiores médias de NL $(4,8 \pm 0,79)$ e a menores médias de NS por endocarpo $(1,6 \pm 0,90)$, ao mesmo tempo, em que, as amostras de CCA apresentaram NL médio de 4,4 $\pm 0,48$ e valor médio de NS de 2,2 $\pm 0,95$. Segundo Sacramento (2009) no interior do endocarpo de taperebá pode conter de dois a cinco lóculos, sem nenhuma ou com até cinco sementes. Em trabalho realizado por Souza et al. (2000) foi verificado que os endocarpos possuem de três a quatro lóculos, contendo de 0 a 3 sementes. Enquanto Pereira (1996) observou até oito lóculos e quatro sementes por endocarpo.

Por apresentar maiores DE e MFE, os frutos oriundos de CPAF-RR apresentaram maior valor médio no RE, variável importante para calcular a necessidade de matéria-prima para a produção de mudas via reprodução sexuada. Em média o RE de CPAF-RR foi de $44,90 \pm 6,77 \%$, enquanto, o de frutos oriundos de CCA foi de $22,49 \pm 5,89 \%$, ambos, abaixo do RE apresentado por Mattietto; Lopes; Menezes (2010), com média de 51,8\%. Inversamente proporcional ao rendimento de endocarpos, constataram-se valores de

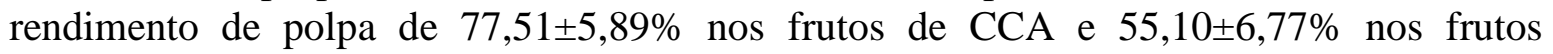
oriundos de CPAF-RR.

Em $1000 \mathrm{~g}$ de frutos teriam em média, 94 frutos, $337 \mathrm{~g}$ de polpa, $663 \mathrm{~g}$ de endocarpos frescos, 430 lóculos e 180 sementes. Para obter $1000 \mathrm{~g}$ de endocarpos frescos são necessários em média 280 frutos ( $2970 \mathrm{~g}$ de amostra). Necessitam-se em média de 53 frutos (561 g) para obter 100 sementes. Para obter 1000 g de polpa são necessários142 frutos, que equivalem a $1510 \mathrm{~g}$ de massa fresca. Observou-se que todas as amostras de frutos avaliadas estavam com SS, pH e AT em conformidade com o padrão de identidade e qualidade para polpas brasileiras (Brasil, 2000). Os resultados obtidos na caracterização físico-química do pericarpo de taperebazeiros das diferentes áreas estudadas estão apresentados na Tabela 2.

Tabela 2. Valores médios de qualidade agroindustrial do pericarpo (epicarpo e mesocarpo) de taperebazeiros cultivados em duas áreas urbanas de Boa Vista, Roraima

\begin{tabular}{|c|c|c|c|c|c|}
\hline \multirow{2}{*}{ Variáveis } & \multicolumn{2}{|c|}{ Áreas } & \multirow{2}{*}{ Valor de $p$} & \multirow{2}{*}{ Média geral } & \multirow{2}{*}{$\mathrm{CV}(\%)$} \\
\hline & CCA & CPAF-RR & & & \\
\hline Sólidos solúveis $\left({ }^{\circ}\right.$ Brix $)$ & $15,4 \pm 0,21$ & $13,4 \pm 0,38$ & $8,799 \mathrm{e}^{-06} *$ & 14,39 & 7,39 \\
\hline Acidez titulável (g de ácido por 100 g) & $1,53 \pm 0,02$ & $1,24 \pm 0,03$ & $7,802 \mathrm{e}^{-08 *}$ & 1,39 & 11,47 \\
\hline $\mathrm{pH}$ & $2,9 \pm 0,02$ & $3,4 \pm 0,02$ & $9,743 \mathrm{e}^{-10 *}$ & 3,2 & 7,72 \\
\hline Relação SS:AT & $10,0 \pm 0,03$ & $10,9 \pm 0,45$ & $1,081 \mathrm{e}^{-3 *}$ & 10,4 & 5,18 \\
\hline Luminosidade & $50,69 \pm 0,66$ & $50,04 \pm 0,67$ & $0,159 \mathrm{~ns}$ & 50,36 & 1,42 \\
\hline Cromaticidade & $45,21 \pm 1,57$ & $35,89 \pm 2,87$ & $2,153 \mathrm{e}^{-3 *}$ & 40,55 & 13,25 \\
\hline Ângulo hue $\left(^{\circ}\right)$ & $71,30 \pm 0,98$ & $67,40 \pm 1,11$ & $3,675 \mathrm{e}^{-3 *}$ & 69,35 & 3,29 \\
\hline
\end{tabular}


$\mathrm{N}=5 ; \mathrm{CCA}=$ Centro de Ciências Agrárias da Universidade Federal de Roraima; CPAF-RR = Centro de Pesquisa Agroflorestal de Roraima; $\mathrm{CV}=$ coeficiente de variação; $*=$ significativo ao nível de $5 \%$ de probabilidade; $n s=$ não significativo ao nível de $5 \%$ de probabilidade.

As polpas de taperebá de CCA são mais doces do que as de CPAF-RR e

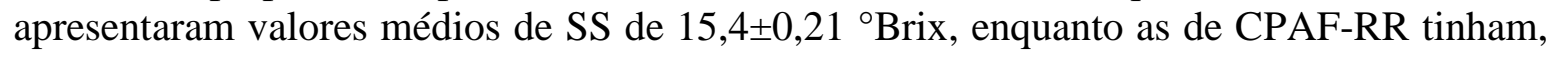
em média, $13,4 \pm 0,38{ }^{\circ}$ Brix. Ambas as médias encontram-se bem acima do mínimo estabelecido por lei, que é 9,0 ${ }^{\circ}$ Brix para sólidos solúveis (Brasil, 2000), e com valores semelhantes aos encontrados na literatura, com resultados variando de 6,86 a 16,3 ${ }^{\circ}$ Brix (Silva et al., 1997; Soares et al., 2006; Mattietto; Lopes; Menezes, 2010; Tiburski et al., 2011; Carvalho et al., 2013; Sousa et al., 2017). É necessário evidenciar que todas as amostras avaliadas, independente da área de coleta, apresentaram valores de SS superiores aos encontrados por Nascimento et al. (2012) em polpas de frutos industrializadas e comercializadas no município de Boa Vista, Roraima, com médias variando de 1,93 a 8,20 ${ }^{\circ}$ Brix.

Os frutos oriundos de CCA são mais ácidos do que os frutos de CPAF-RR, apresentando AT média de 1,53 $\pm 0,02 \mathrm{~g}$ de ácido $100 \mathrm{~g}^{-1}$ de amostra, enquanto, os frutos colhidos em CPAF-RR apresentaram acidez titulável média de 1,23 $\pm 0,02 \mathrm{~g}$ de ácido $100 \mathrm{~g}^{-1}$ de amostra. Os valores médios de AT em ambas as áreas são semelhantes aos registrados na literatura, com valores variando de 0,89 a 1,40 (Carvalho et al., 2013; Sousa et al., 2017). Os valores aqui registrados são inferiores aos registrados por Mattietto; Lopes; Menezes (2010) em frutos de Belém, Pará, e de Sanches et al. (2017) em Alta Mira, Pará, com médias de 1,86 e 1,94 g de ácido $100 \mathrm{~g}^{-1}$ de amostra, respectivamente. Ressalta-se que os valores de acidez titulável de frutos de CCA e CPAF-RR são superiores aos encontrados em polpas de frutos industrializadas e comercializadas no município de Boa Vista, Roraima, com valores médios variando de 0,69 a $1,31 \mathrm{~g}$ de ácido $100 \mathrm{~g}^{-1}$ de amostra (Nascimento et al., 2012).

Na relação SS:AT, indicador de maturação, a diferença entre as polpas de taperebá cultivadas em CCA e CPAF-RR também foi constatada. O maior valor médio foi registrado

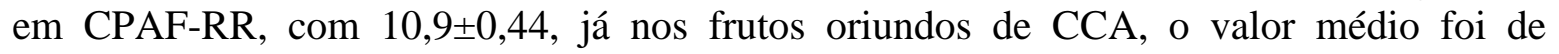
$10,0 \pm 0,01$, semelhantes aos valores encontrados na literatura, que vão de 10,20 a 11,24 (Soares et al., 2006; Tiburski et al., 2011; Sousa et al., 2017). As amostras coletadas em CCA e CPAF apresentam valores de relação SS:AT superiores aos encontrados em feiras livres de Belém, Pará, com média geral de 5,43 (Mattietto; Lopes; Menezes, 2010) e em polpas de frutos industrializadas e comercializadas em Boa Vista, Roraima, com média geral de 5,77 (Nascimento et al., 2012).

Constataram-se que as polpas dos frutos de taperebazeiros avaliadas são, no geral, ácidas, com pH médio de 3,2. Os frutos oriundos de CCA são mais ácidos que os frutos de

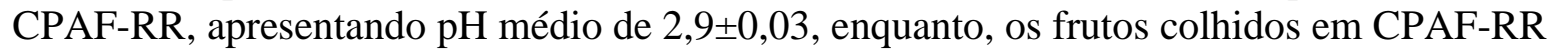
apresentaram valores médios de $\mathrm{pH}$ de $3,37 \pm 0,01$, representando menor concentração de íons $\mathrm{H}^{+}$e maior concentração de íons $\mathrm{OH}^{-}$em CPAF-RR. Os valores médios de $\mathrm{pH}$ das 
áreas analisadas assemelham-se aos registrados na literatura, com valores variando de 2,26 a 3,45 (Dias; Schwan; Lima, 2003; Tiburski et al., 2011; Carvalho et al., 2013; Santos; Figueiredo Neto; Donzeli, 2016; Silva et al. 2017; Sousa et al., 2017).

A luminosidade do pericarpo, que indica se o produto é mais claro ou mais escuro, não apresentou diferença significativa ao nível de $5 \%$ de probabilidade $(\mathrm{p}=0,159)$ entre os frutos das áreas estudadas. Os frutos de taperebazeiros de CCA e CPAF-RR apresentaram L* média de 50,36, indicando luminosidade moderada. Os pericarpos de frutos colhidos para o trabalho são mais claros que as polpas de taperebá comercializadas em Mossoró, Rio Grande do Norte, avaliadas por Sousa et al. (2017), com valores entre 35,10 e 47,21, e mais escuros do que os registros de Mattietto; Lopes; Menezes (2010) em Belém, Pará, de frutos com luminosidade média de 61,02.

As polpas dos frutos colhidos em CCA apresentaram valores de $\mathrm{C}^{*}$ (indicador da intensidade do tom) superiores aos registrados nas polpas de frutos colhidos em CPAF-RR, indicando coloração mais intensa. Os valores médios de $\mathrm{C}^{*}$ para as polpas de frutos de

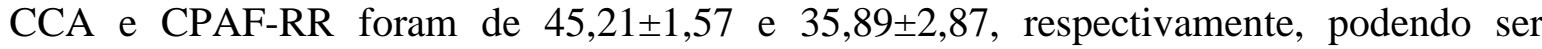
considerados valores médios de cromaticidade. Santos; Figueiredo; Donzeli (2016) avaliando a qualidade de polpas de quatro marcas diferentes, comercializadas nas cidades de Juazeiro, Bahia e Petrolina, em Pernambuco, registraram valores de cromaticidade variando de 14,12 a 19,24 , que é bem menor do que a média geral $(40,55)$ aferida nas amostras de CCA e CPAF. Valores de cromaticidade baixos também foram registrados por Silva et al. (2018) em avaliação de maturação de frutos de taperebazeiros cultivados em Distrito de Hidrolândia, Goiás, com cromaticidade média de frutos maturos de 25,86.

Apesar de os frutos de taperebazeiros oriundos de CCA e CPAF-RR avaliados nesta pesquisa apresentarem diferença estatística significativa na variável ângulo hue (indicador de tonalidade), é valido ressaltar que ambos são considerados de coloração e tonalidade amarelo alaranjado, que varia no sistema CIE $\mathrm{L}^{*} \mathrm{a}^{*} \mathrm{~b}^{*}$ de 50 a $90^{\circ}$ para ângulo hue. Os valores médios de ângulo hue aferidos em CCA de 71,30 $\pm 0,98^{\circ}$ e em CPAF-RR de $67,40 \pm 1,11^{\circ}$ estão dentro da faixa de valores médios encontrados na literatura para frutos e polpas de taperebazeiros, que variam de 66,08 a 88,80 ${ }^{\circ}$ (Santos; Figueiredo Neto; Donzeli, 2016; Silva et al., 2018).

Acredita-se que essas variações entre as duas áreas podem ser relacionadas às condições de manejo e diferenças genéticas entre as matrizes, não registradas oficialmente na ocasião desta pesquisa. No entanto, de maneira geral, os frutos aqui estudados são semelhantes aos encontrados na literatura, apresentando excelentes possibilidades para a seleção e melhoramento genético.

\section{CONSIDERAÇÕES FINAIS}

Há diferença significativa $(\mathrm{p}<0,05)$ na qualidade física e físico-química de frutos de taperebazeiros cultivados em diferentes áreas urbanas de Boa Vista, Roraima. 
Os frutos e endocarpos de taperebazeiros têm formatos obovóides, ovóides e elípticos. As polpas de taperebazeiros avaliadas estão em conformidade com os padrões de identidade e qualidade para polpa de cajá, apresentando ótima qualidade e bom rendimento, com valores desejáveis para agroindústria.

\section{AGRADECIMENTOS}

Ao apoio da Empresa Brasileira de Pesquisa Agropecuária (Embrapa Roraima) e ao Conselho Nacional de Desenvolvimento Científico e Tecnológico.

\section{REFERÊNCIAS}

AZEVEDO, D. M.; MENDES, A. M.; FIGUEIREDO, A. F. Característica da germinação e morfologia do endocarpo e plântula de taperebá (Spondias mombin L.) - Anarcadiaceae. Revista Brasileira de Fruticultura, Jaboticabal, v. 26, n. 3, p. 534 - 537, 2004.

BRASIL. Instrução Normativa $\mathrm{n}^{\circ}$. 1, de 7 de jan de 2000. Regulamento técnico geral para fixação dos padrões de identidade e qualidade para polpa de fruta. D.O.U., n 6 , Brasília, 10 de jan de 2000, Seção I., p.54-58.

CAMPOS, G. M. Estatística prática para docentes e pós-graduandos. São Paulo, 2000. 59p.

CARVAlHO, J. M. de; MAIA, G. A.; FONSECA, A. V. V. da; SOUSA, P. H. M. de; RODRIGUES, S. Effect of processing on physicochemical composition, bioactive compounds and enzymatic activity of yellow mombin (Spondias mombin L.) tropical juice. Journal of Food Science and Technology, Illinois, v. 52, n. 2, p. 1182 - 1187, 2013.

CUNHA, A.C.; SILVA, R.A.; PEREIRA, J.D.B.; SANTOS, R.S. Efeito da espessura da polpa, tamanho e peso de frutos de taperebá (Spondias mombin L.) sobre o parasitismo natural (Hymenoptera: Braconidae) em moscas-das-frutas (Diptera: Tephritidae). Revista de Agricultura, Piracicaba, v. 86, n. 2, p. 125 - 133, 2011.

DIAS, D. R.; SCHWAN, R. F.; LIMA, L. C. O. Metodologia para elaboração de fermentado de cajá (Spondias mombin L.). Ciência e Tecnologia de Alimentos, Campinas, v. 23, n. 3, p. 342 - 350, 2003.

DUARTE, O. R.; SCHWENBER, D. R.; OLIVEIRA, J. M. F. de; CHAGAS, E. A.; ALBUQUERQUE, T. C. S. Ocorrência e distribuição geográfica do taperebá (Spondias mombin L.) em Roraima. In: Congresso brasileiro de fruticultura, 21. 2010, Natal. Frutas: saúde, inovação e responsabilidade. p.01-04.

INSTITUTO ADOLFO LUTZ (IAL). Métodos físico-químicos para análise de alimentos. São Paulo: Instituto Adolfo Lutz, 2008. 1020 p. CDD 614.028. 
MATTIETTO, R A.; LOPES, A. S.; MENEZES, H. C. Caracterização física e físicoquímica dos frutos da cajazeira (Spondias mombin L.) e de suas polpas obtidas por dois tipos de extrator. Brazilian Journal Food Technology, Campinas, v. 13, n. 3, p. 156 - 164, 2010.

MOURA, F. T.; SILVA, S. M.; MARTINS, L. P.; MENDONÇA, R. M. N.; ALVES, R. E.; FILGUEIRAS, H. A. C. Evolução do crescimento e da maturação de frutos de cajazeira (Spondias mombin L.). Proceedings of the Interamerican Society for Tropical Horticulture - Fruits/Frutales, Homestead, v. 47, p. 231 - 233, 2003.

NASCIMENTO, C. R.; NEVES, L. C.; GRÍGIO, M. L.; CAMPOS, A. J. de.; CHAGAS, E. A.; SOUZA, A. de A. Avaliação da qualidade de polpas de frutos industrializadas e comercializadas no município de Boa Vista - RR. Revista Agro@mbiente On-line, Boa Vista, v. 6, n. 3, p. 263-267, 2012.

PEREIRA, K. S. N. Cajá (Spondias mombin L.): características da unidade de dispersão, propagação sexuada e assexuada. Areia: UFPB, 1996. 31p.

PIMENTEL-GOMES, F. Curso de estatística experimental. Piracicaba: FEALQ, 2009, $451 \mathrm{p}$.

QUADROS, B. R. de; FELIPE, j. P.; CALDAS, I. G. R.; FERREIRA, G.; NASCIMENTO, W. M. O. do; CAVARIANI, C. Superação de dormência em sementes de taperebá (Spondias mombin L., Anacardiaceae). In: Reunião Anual da SBPC. 64. São Luíz, 2012, p.01-02.

R CORE TEAM. R: A language and environment for statistical computing. Vienna, Austria: R Foundation for Statistical Computing, 2018. Disponível em: https://www.rproject.org/.

SOARES, E. B.; GOMES, R. L. F.; CARNEIRO, J. G. M.; NASCIMENTO, F. N.; SILVA, I. C. V.; COSTA, J. C. L. Caracterização física e química de frutos de cajazeira. Revista Brasileira de Fruticultura, Jaboticabal, v. 28, n. 3, p. 518 - 519, 2006.

SACRAMENTO, C. K.; SOUZA, F. X. Cajá. In: SEREJO, J. A. dos S. et al (Ed.). Fruticultura tropical: espécies regionais e exóticas. Brasília: Embrapa Informação Tecnológica, 2009. 5. p.83-105.

SANCHES, A. G.; SILVA, M. B.; MOREIRA, E. G. S.; COSTA, J. M. 1Metilciclopropeno na manutenção da qualidade pós-colheita de taperebá (Spondia mombin L.). Revista de Agricultura Neotropical, Cassilândia, v. 4, n. 2, p. 56 - 62, 2017.

SANTOS, E. H. F.; FIGUEIREDO NETO, A.; DONZELI, V. P. Aspectos físico-químicos e microbiológicos de polpas de frutas comercializadas em Petrolina (PE) e Juazeiro (BA). Brazilian Journal of Food Technology, Campinas, v. 19, e2015089, p. 01 - 09, 2016. 
SILVA, A. de P. V.; MAIA, G. A.; OLIVEIRA, G. S. F. de; FIGUEIREDO, R. W. de; BRASIL, I. M. Características de qualidade do suco polposo de cajá (Spondias lutea L.) obtido por extração mecânico-enzimática. Ciência e Tecnologia de Alimentos, Campinas, v. 17, n. 3, p. 233 - 236, 1997.

SILVA, T. L. L.; SILVA, E. P. da.; ASQUIERI, E. R.; VIEIRA, E. C. S.; SILVA, J. S.; SILVA, F. A. da.; DAMIANI, C. Physicochemical characterization and behavior of biocompounds of caja-manga fruit (Spondias mombin L.). Food Science and Technology, Campinas, v. 23, n. 3, p. 399 - 406, 2018.

SOUSA, A. S. de; SOARES, K. M. de P.; GÓIS, V. A. de; FREIRE, B. C. F. Qualidade microbiológica e físico-química de polpas de umbu-cajá e cajá comercializadas em Mossoró, Higiene Alimentar, São Paulo, v. 31, p. 42 - 46, 2017.

SOUZA, F. X.; SOUSA, F. H. L.; FREITAS, J. B. S.; ROSSETTI, A. G. Aspectos morfológicos da unidade de dispersão de cajazeira. Pesquisa Agropecuária Brasileira, Brasília, v. 35, n. 1, p. 215 - 220, 2000.

TIBURSKI, J. H.; ROSENTHAL, A.; DELIZA, R.; GODOY, R. L. de O.; PACHECO, S. Nutritional properties of yellow mombin (Spondias mombin L.) pulp. Food Research International, Ontario, v. 44, p. 2326 - 2331, 2011. 